\title{
Megamaps: Construction and Examples
}

\author{
Alexander Zvonkin \\ LaBRI, Université Bordeaux I, 351 cours de la Libération, F-33405 Talence Cedex France \\ E-mail: zvonkin@labri.u-bordeaux.fr
}

received February 3, 2001, revised April 10, 2001, accepted April 16, 2001.

\begin{abstract}
We consider the usual model of hypermaps or, equivalently, bipartite maps, represented by pairs of permutations that act transitively on a set of edges $E$. The specific feature of our construction is the fact that the elements of $E$ are themselves (or are labelled by) rather complicated combinatorial objects, namely, the 4-constellations, while the permutations defining the hypermap originate from an action of the Hurwitz braid group on these 4-constellations. The motivation for the whole construction is the combinatorial representation of the parameter space of the ramified coverings of the Riemann sphere having four ramification points.
\end{abstract}

Keywords: Riemann surface; ramified covering; dessins d'enfants; Belyi function; braid group; Hurwitz scheme

\section{Introduction}

In the well-known combinatorial approach to maps or hypermaps drawn on two-dimensional surfaces the maps are represented as pairs of permutations on a set $E$ of the edges (each edge connects the verticies of two different sorts, which we color black and white); see, for example, [CM92]. The edges are usually interpreted geometrically as segments of curves drawn on a surface. However, one of the advantages of the permutation model is its abstract nature. Namely, one may take as the set $E$ an arbitrary finite set, and introduce the permutations acting on $E$ by using the particular structure of the elements of this set.

In this paper, the elements of $E$ will be the 4-constellations, that is, the sequences of permutations $\left[g_{1}, g_{2}, g_{3}, g_{4}\right], g_{i} \in S_{n}$, acting transitively on the underlying set of $n$ elements, and such that the product $g_{1} g_{2} g_{3} g_{4}=$ id. The permutations that will act on the set $E$ itself originate from an action of the Hurwitz braid group on the 4-constellations; they will be introduced in Section 3.2.

The aim of the whole construction is to represent a parameter space of the ramified coverings of the Riemann sphere with four ramification points. When the number of the ramification points is three, the corresponding coverings may be considered as a kind of "isolated points"; such coverings are studied by the theory of dessins d'enfants [Sch94]. At the same time the coverings with four ramification points depend on one complex parameter. But this parameter is not just a complex number: it "lives" on a Riemann surface, and this surface in its turn may be described by a dessin d'enfant.

This construction is known in the inverse Galois theory as a Hurwitz scheme. But it is a very difficult task to extract its combinatorial nature from the corresponding publications. We cite here only few papers which are close to our proper interests, namely, to the dessins d'enfants: [Fri90], [Cou97], [Gra96]. In our paper we introduce the combinatorial construction, clarify its relations with the ramified coverings and give several examples.

1365-8050 @ 2001 Maison de l'Informatique et des Mathématiques Discrètes (MIMD), Paris, France 


\section{Constructing ramified coverings}

\subsection{Riemann's existence theorem}

One of the most wide spread methods of dealing with Riemann surfaces is to represent them as ramified coverings of the complex Riemann sphere $\overline{\mathbf{C}}=\mathbf{C} \cup\{\infty\}$. Let $\mathbf{X}$ be a compact Riemann surface, and $f: \mathbf{X} \rightarrow \overline{\mathbf{C}}$ a non-constant meromorphic function. Then there exists a finite set $Y \subset \overline{\mathbf{C}}, Y=\left\{y_{1}, \ldots, y_{k}\right\}$ such that for any $y \in \overline{\mathbf{C}} \backslash Y$ the preimage $f^{-1}(y)$ has the same cardinality $n=\left|f^{-1}(y)\right|=\operatorname{deg} f$, while for the $y_{i} \in Y, i=1, \ldots, k$ we have $\left|f^{-1}\left(y_{i}\right)\right|<n$. The elements of $Y$ are called the critical values of $f$, or the ramification points of the covering $f: \mathbf{X} \rightarrow \overline{\mathbf{C}}$. Sometimes, for purely technical reasons, it may be convenient to add to the set $Y$ a certain number of generic (that is, non critical) values. We fix a numbering of the elements of $Y$ and will consider it as a sequence of points in $\overline{\mathbf{C}}$ rather than a set; we write sequences in square brackets: $\left[y_{1}, \ldots, y_{k}\right]$.

Let $y_{0} \in \overline{\mathbf{C}} \backslash Y$ be a generic value of $f$. Denote $E=f^{-1}\left(y_{0}\right),|E|=n$. Let us draw $k$ oriented paths $\gamma_{i}$ from $y_{0}$ to $y_{i}, i=1, \ldots, k$ on the sphere $\overline{\mathbf{C}}$ in such a way that (i) the paths do not intersect outside $y_{0}$; and (ii) in the small vicinity of $y_{0}$ the order $\gamma_{1}, \ldots, \gamma_{k}$ of the paths corresponds to their counter-clockwise order around $y_{0}$. The path $\gamma_{i}$ taken in the opposite direction (from $y_{i}$ to $y_{0}$ ) is denoted by $\gamma_{i}^{-1}$. Let us now transform each $\gamma_{i}$ into a lacet $l_{i}$ belonging to the fundamental group $\pi_{1}\left(\overline{\mathbf{C}} \backslash Y, y_{0}\right)$, which first leaves $y_{0}$ and goes along the path $\gamma_{i}$ until it comes to a small neighborhood of $y_{i}$; then it makes a complete counter-clockwise turn around $y_{i}$ and returns to $y_{0}$ along $\gamma_{i}^{-1}$. The lifting of $l_{i}$ under $f$, that is, its preimage $f^{-1}\left(l_{i}\right)$, is a set of $n$ oriented paths in $\mathbf{X}$ which don't intersect outside $E$; each of them leads from a point $x \in E$ to another point $x^{\prime} \in E$. As the procedure is entirely reversible (the lifting of the lacet $l_{i}^{-1}$ leads from $x^{\prime}$ to $x$ ), this gives us a permutation on the set $E$. We denote this permutation as $g_{i}$. The group $G=\left\langle g_{1}, \ldots, g_{k}\right\rangle$ is the monodromy group of the covering. The correspondence $l_{i} \mapsto g_{i}$ generates a surjective group homomorphism $\pi_{1}\left(\overline{\mathbf{C}} \backslash Y, y_{0}\right) \rightarrow G$.

The sequence of permutations $\left[g_{1}, \ldots, g_{k}\right]$ satisfies the following conditions. First, the group $G$ acts transitively on $E$; this means that the corresponding Riemann surface is connected. Second, the product $g_{1} \cdot \ldots \cdot g_{k}=$ id. This relation reflects the fact that the product of the lacets $l_{1} \cdot \ldots \cdot l_{k} \in \pi_{1}\left(\overline{\mathbf{C}} \backslash Y, y_{0}\right)$ goes around all the points $y_{1}, \ldots, y_{k}$, and, taking into account that we are on the sphere, the resulting lacet can be retracted to the point $y_{0}$.

Definition 2.1 (Constellation) A sequence of permutations $\left[g_{1}, \ldots, g_{k}\right], g_{i} \in S_{n}$ which acts transitively on $n$ points and such that the product $g_{1} \cdot \ldots \cdot g_{k}=\mathrm{id}$, is called a constellation (or a $k$-constellation). The passport of a constellation is the sequence $\left[\lambda^{(1)}, \ldots, \lambda^{(k)}\right]$ of partitions $\lambda^{(i)} \vdash n$, where $\lambda^{(i)}$ is the cyclic structure of $g_{i}$. Two constellations $\left[g_{1}, \ldots, g_{k}\right]$ and $\left[g_{1}^{\prime}, \ldots, g_{k}^{\prime}\right]$ are isomorphic if there exists an $h \in S_{n}$ such that $g_{i}^{\prime}=h^{-1} g_{i} h$ for $i=1, \ldots, k$.

The motivation for the term "constellation" is that the paths $\gamma_{i}$ form a star graph on the sphere, with the center in $y_{0}$. The preimage of this graph under $f$ is a "collection of stars" connected to one another.

Theorem 2.2 (Riemann's existence theorem) For any finite sequence $Y=\left[y_{1}, \ldots, y_{k}\right] \subset \overline{\mathbf{C}}$ and for any constellation $\left[g_{1}, \ldots, g_{k}\right], g_{i} \in S_{n}$, there exists a compact Riemann surface $\mathbf{X}$ and a meromorphic function $f: \mathbf{X} \rightarrow \overline{\mathbf{C}}$ such that $y_{1}, \ldots, y_{k}$ are the critical values of $f$, and $g_{1}, \ldots, g_{k}$ the corresponding permutations. The covering $f: \mathbf{X} \rightarrow \overline{\mathbf{C}}$ is unique up to an isomorphism of $\mathbf{X}$.

One of the most remarkable features of this theorem is the fact that there are no conditions on the critical values $y_{1}, \ldots, y_{k}$ : they may be chosen arbitrarily. A proof may be found, for example, in [Vö196]. 


\section{2 "Dessins d'enfants" and hypermaps}

Let us summarize once more what data one must supply in order to represent a ramified covering of the sphere $\overline{\mathbf{C}}$ by a Riemann surface $\mathbf{X}$. It consists of (i) a constellation $\left[g_{1}, \ldots, g_{k}\right]$, which is a discrete, or combinatorial part of information, and (ii) a sequence of $k$ continuous parameters (or "moduli") $y_{1}, \ldots, y_{k} \in \overline{\mathbf{C}}$.

We may consider various equivalence relations on coverings, depending on the goal of a particular research. For our goal, we will consider as equivalent those coverings that may be obtained from one another by a complex isomorphism of the sphere $\overline{\mathbf{C}}$. Such an equivalence preserves the complex structure on $\mathbf{X}$. Recall that the complex isomorphisms of $\overline{\mathbf{C}}$ are the linear fractional transformations. Choosing an appropriate linear fractional transformation, we may take any three points among $y_{1}, \ldots, y_{k}$, for example, $y_{k-2}, y_{k-1}, y_{k}$, and move them to any three prescribed positions fixed in advance, for example, to 0,1 and $\infty$; this choice fixes the linear fractional transformation. There remain $k-3$ continuous parameters $z_{1}, \ldots, z_{k-3}$ instead of $k$ : they are the images of $y_{1}, \ldots, y_{k-3}$ under the linear fractional transformation. Changing "a little" the parameters $z_{1}, \ldots, z_{k-3}$ one changes "a little" the Riemann surface $\mathbf{X}$ together with its complex structure.

Now the question arises:

\section{What if $k=3$ ?}

In this case all the continuous parameters dissapear, and the only data that remain are combinatorial, namely, the triple of permutations $\left[g_{1}, g_{2}, g_{3}\right]$, representing the ramifications over 0,1 and $\infty$. (The constellation in question may be thought of merely as a pair of permutations, because the third permutation is determined by the relation $g_{1} g_{2} g_{3}=$ id.) The whole construction suddenly becomes rigid: we cannot change it "a little" any more. We would like to emphasize once more that the object thus represented is a Riemann surface, that is, a one-dimensional complex analytic variety, which is a much more complicated structure than that of just a topological two-dimensional variety.

One may ask if any Riemann surface $\mathbf{X}$ may be represented this way? The answer is no, but the class of the surfaces so representable is probably the most interesting one: namely, it is the class of the arithmetic surfaces, as is asserted by the now famous Belyi theorem [Bel79]:

Theorem 2.3 (Belyi theorem) The Riemann surface $\mathbf{X}$ is defined over the field $\overline{\mathbf{Q}}$ of algebraic numbers if and only if there exists a covering $f: \mathbf{X} \rightarrow \overline{\mathbf{C}}$ unramified outside 0,1 and $\infty$.

A function $f$ unramified outside 0,1 and $\infty$ is called Belyi function, and a pair $(\mathbf{X}, f)$ where $f$ is a Belyi function on $\mathbf{X}$ is called Belyi pair. The phrase "defined over $\overline{\mathbf{Q}}$ " means that $\mathbf{X}$ may be represented as an algebraic curve $F(x, y)=0$ such that the coefficients of $F$ are algebraic numbers. In such a case the coefficients of $f$ (as a function of $x$ and $y$ ) may also be chosen as algebraic numbers. This is the framework of the so-called theory of dessins d'enfants ("children's drawings" in French), a term coined by Grothendieck [Gro84]. Grothendieck made a remark that for a Belyi function $f$ the set $f^{-1}([0,1]) \subset \mathbf{X}$ is a graph, or rather a bipartite map drawn on the surface X. It has two sorts of vertices: the "black" ones which are the preimages of 0 , and the "white" ones which are the preimages of 1 , the degree of each vertex being equal to the multiplicity of the corresponding root of $f=0$ or $f=1$. And inside each face of this map there is exactly one pole of $f$, its multiplicity being equal to the degree of the face.

In fact, the corresponding combinatorial object, that is, the triple of permutations, is very well known under the name of a hypermap and was actively studied for many years in combinatorics: see, for example, a review [CM92]. According to the tradition we use the notation $[\sigma, \alpha, \varphi]$ instead of $\left[g_{1}, g_{2}, g_{3}\right]$. 
Definition 2.4 (Hypermap) A hypermap is a triple of permutations $[\sigma, \alpha, \varphi]$ which act transitively on a finite set $E$ and which satisfy the relation $\sigma \alpha \varphi=$ id.

Thus a hypermap is nothing else but a 3-constellation. The third permutation $\varphi$ is often omitted from the definition, as $\varphi=\alpha^{-1} \sigma^{-1}$. The greek letters $\sigma, \alpha$ and $\varphi$ were chosen as the "first letters" of the French terms "sommet" (vertex), "arête" (edge) and "face" (face).

Example 2.5 The hypermap shown in Figure 1 has 12 edges. The triple of permutations representing this hypermap is

$$
\begin{aligned}
& \sigma=(1,2,3,4,5)(6,7,8,9)(10,11,12), \\
& \alpha=(2,8,10)(5,12,9), \\
& \varphi=(1,5,8)(2,12,4,3)(6,9,11,10,7) .
\end{aligned}
$$

The permutation $\sigma$ shows the cyclic order of the edges around the black vertices (in counter-clockwise direction); $\alpha$ gives the similar information for the white vertices. If we use a convention to always write the labels of the edges on the left side while going from black to white, then all the labels corresponding to a face (that is, to a cycle of $\varphi$ ) lie inside the corresponding face (see figure). This time the labels are also taken in counter-clockwise direction for all the faces except the outer one; the outer face (in the planar case) should be looked at from the opposite side of the globe. There is a tradition to call the cycles of $\alpha$ hyperedges, and to call the white vertices their middle points. When all the white vertices are of degree 2 , they are often erased, and the resulting image becomes a usual map with only one sort of vertices and without any bipartite structure.

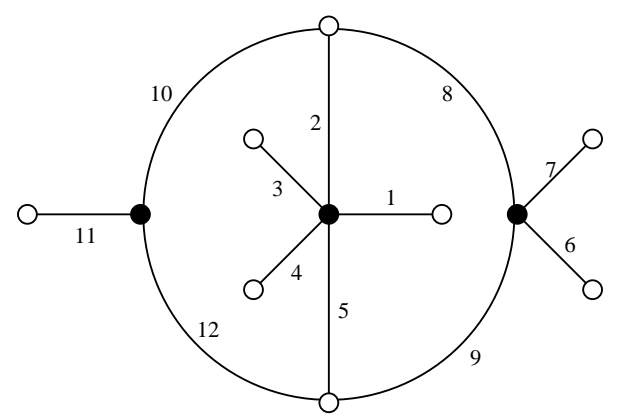

Fig. 1: A hypermap

It is a remarkable fact that such a simple object as just a pair of permutations $\sigma$ and $\alpha$ posesses an incredibly rich variety of mathematical structures: the complex structure of the corresponding Riemann surface $\mathbf{X}$, a number field (the field of definition of the Belyi pair $(\mathbf{X}, f)$ ), its Galois group, etc. A hypermap, whether we look at it as at a triple of permutations or as at a picture (a geometric objetc), should be considered as one of the legitimate ways of representing the corresponding covering $f: \mathbf{X} \rightarrow \overline{\mathbf{C}}$ with only three ramification points 0,1 and $\infty$.

But the goal of this paper is to study the coverings not with three but with four ramification points. 


\section{Coverings with four ramification points}

\subsection{A space of coverings}

As we have seen in Section 2, in order to represent a covering with four ramification points we must supply the following data: (i) a 4-constellation $C=\left[g_{1}, g_{2}, g_{3}, g_{4}\right]$, and (ii) four ramification points, which may be chosen as $0, z, 1$ and $\infty$. Here $z$ is an arbitrary complex number different from 0,1 and $\infty$. We take the ramification points $0, z, 1, \infty$ in this specific order because later on it will be convenient to take $z$ in the segment $[0,1]$.

This time there is no hope that in general such a covering will be defined over $\overline{\mathbf{Q}}$, because the value of $z$ may well be transcendental. But the object we are interested in is not a specific covering but the space of all such coverings. In a way, we would like to parameterize this space.

Let us fix a passport $\pi$ of a 4-constellation, and consider the set of all the pairs $\{(C, z)\}$, where $C$ is a constellation with the passport $\pi$. Denote this set $\mathcal{M}$.

Proposition 3.1 The set $\mathcal{M}$ has a structure of a (non-compact and probably not connected) Riemann surface.

Proof. The number of points above any fixed value of $z$ is always the same, namely, it is the number of non-isomorphic constellations with the passport $\pi$. The complex structure in the neighborhood of a point $(C, z)$ may be introduced by taking as a coordinate the $z$ itself.

We may compactify $\mathcal{M}$ by using the ordinary procedure described in any textbook on Riemann surfaces, namely, by adding the necessary number of points above $z=0,1, \infty$. Let us take a connected component of the resulting compact Riemann surface; we denote it also by $\mathcal{M}$. A remarkable fact is that $\mathcal{M}$ is already supplied with a Belyi function (and is therefore defined over $\overline{\mathbf{Q}}$ )!

Proposition 3.2 The function $(C, z) \mapsto z$ is a Belyi function on $\mathcal{M}$.

Proof. By construction, any value of $z \neq 0,1, \infty$ is not critical because it has the same number of preimages $(C, z)$.

Now our natural goal is to find the triple of permutations that describes the 'dessin d'enfant' which corresponds to the Belyi function

$$
f: \mathcal{M} \rightarrow \overline{\mathbf{C}}:(C, z) \mapsto z
$$

In order to do that we must understand what happens when the parameter $z$ starts at a particular base point $z_{0}$, goes around 0 , or 1 , or $\infty$, and returns to $z_{0}$. It is natural to take $z_{0} \in[0,1]$ : then the permutations we are looking for will combinatorially describe the hypermap drawn on $\mathcal{M}$ which is the preimage of the segment $[0,1]$, as it should be according to our convention.

\subsection{Hurwitz braid group}

The Hurwitz braid group, or the sphere braid group $\mathcal{H}_{k}$ with $k$ strings has $k-1$ generators $\sigma_{1}, \ldots, \sigma_{k-1}$ and the following relations:
(a) $\sigma_{i} \sigma_{j}=\sigma_{j} \sigma_{i}$ for $|i-j| \geq 2$;
(b) $\sigma_{i} \sigma_{i+1} \sigma_{i}=\sigma_{i+1} \sigma_{i} \sigma_{i+1}$ for $i=1, \ldots, k-2$;
(c) $\sigma_{1} \sigma_{2} \ldots \sigma_{k-2} \sigma_{k-1}^{2} \sigma_{k-2} \ldots \sigma_{2} \sigma_{1}=\mathrm{id}$. 
The usual, or plane braid group $\mathcal{B}_{k}$ has the same generators but only the relations (a) and (b), see [Bir74]. Its elements describe the movements of the 'configurations' of $k$ distinct points on the plane. If these points happen to live on the sphere, there is an additional relation (c). Below we illustrate this relation, see Figure 2: one may see that the trajectory of the movement of the first point is not contractible on the plane, but is on the sphere.
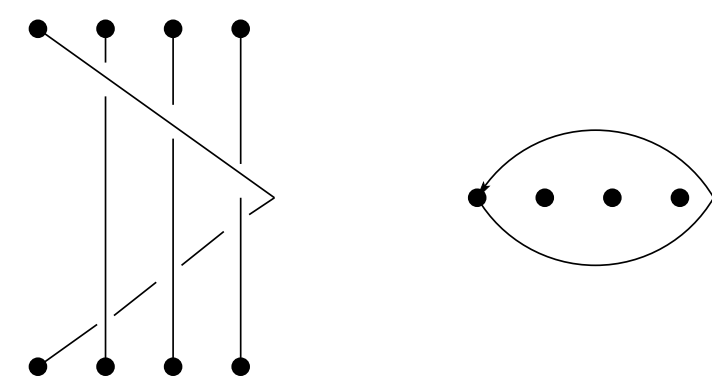

Fig. 2: The braid of the relation (c) and the same braid seen "from the above"

The Hurwitz braid group $\mathcal{H}_{k}$ acts on the isomorphism classes of $k$-constellations in the following way:

$$
\sigma_{i}: g_{i} \mapsto g_{i}^{\prime}=g_{i+1}, \quad g_{i+1} \mapsto g_{i+1}^{\prime}=g_{i+1}^{-1} g_{i} g_{i+1}, \quad \text { and } \quad g_{j} \mapsto g_{j}^{\prime}=g_{j} \quad \text { for } \quad j \neq i, i+1 .
$$

This transformation obviously preserves the product $g_{1} \cdot \ldots \cdot g_{k}$. It is easy to verify the above relations (a), (b), (c) by direct computation. For example, the element $\sigma_{1} \sigma_{2} \ldots \sigma_{k-2} \sigma_{k-1}^{2} \sigma_{k-2} \ldots \sigma_{2} \sigma_{1}$ conjugates each of the $g_{i}$ by $g_{1}$; the result is thus an isomoprphic constellation.

The geometric meaning of the operation performed by $\sigma_{i}$ is very simple. It means that the neighboring critical values $y_{i}$ and $y_{i+1}$ in the sequence $\left[y_{1}, \ldots, y_{k}\right]$ have exchanged their places in the sequence. (We would probably prefer to just transpose $g_{i}$ and $g_{i+1}$ in the constellation; but then the product may have changed.) These operations were introduced, with exactly this aim, by Hurwitz in 1891 [Hur1891], which was 34 years before the introduction of the braid groups themselves by E. Artin.

Now everything is ready to see what happens when $z$ goes around 0,1 and $\infty$. We work inside the group $\mathcal{H}_{4}$ with four strings. The corresponding lacets are shown in Figure 3. Their representation in the form of braids is as follows: the lacet that turns around 0 is equal to $\Sigma=\sigma_{1}^{2}$; the one that turns around 1 is equal to $A=\sigma_{2}^{2}$; finally, the third lacet that turns around infinity is equal to $\Phi=\sigma_{2}^{-1} \sigma_{3}^{2} \sigma_{2}$.

Lemma 3.3 In the Hurwitz braid group $\mathcal{H}_{4}$ the product $\Sigma \mathrm{A} \Phi=\mathrm{id}$.

Proof. We have $\Sigma A \Phi=\sigma_{1}^{2} \sigma_{2} \sigma_{3}^{2} \sigma_{2}$. Conjugating this element by $\sigma_{1}$ we get

$$
\sigma_{1}^{-1}\left(\sigma_{1}^{2} \sigma_{2} \sigma_{3}^{2} \sigma_{2}\right) \sigma_{1}=\sigma_{1} \sigma_{2} \sigma_{3}^{2} \sigma_{2} \sigma_{1}=\text { id }
$$

according to the relation (c). One might also directly verify that the action of the product $\Sigma A \Phi$ on a constellation $\left[g_{1}, g_{2}, g_{3}, g_{4}\right]$ conjugates all the $g_{i}$ by $g_{2}$ and thus gives an isomorphic constellation.

Remark 3.4 All the three operations $\Sigma$, A and $\Phi$ preserve the passport of a constellation. One of the consequences of this is the fact that all the orbits of the group $\mathcal{P}=\langle\Sigma, \mathrm{A}, \Phi\rangle\left\langle\mathcal{H}_{4}\right.$ are finite. It is clear that 
in order to get a connected hypermap one must take on orbit of $\mathcal{P}$ together with the three permutations $\Sigma, \mathrm{A}, \Phi$ acting on it. It is this object that we call megamap.
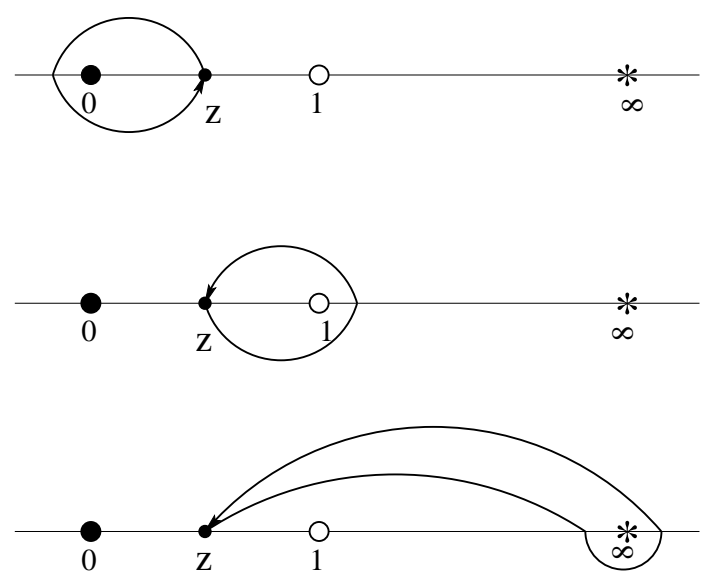

Fig. 3: The fourth critical value $z$ starts at a point in the segment $[0,1]$ and makes a complete turn around 0,1 or $\infty$ in the counter-clockwise direction

\subsection{Megamaps}

Here we summarize the definition of a megamap.

Definition 3.5 A megamap is a set $\mathbf{E}$ and a triple of permutations $[\Sigma, \mathrm{A}, \Phi]$ acting on $\mathbf{E}$, where:

1) The elements of $\mathbf{E}$ are the isomorphism classes of 4-constellations $\left[g_{1}, g_{2}, g_{3}, g_{4}\right], g_{i} \in S_{n}$.

1) The set $\mathbf{E}$ itself is an orbit of the subgroup $\mathcal{P}=\langle\Sigma, \mathrm{A}, \Phi\rangle\left\langle\mathcal{H}_{4}\right.$ of the Hurwitz braid group $\mathcal{H}_{4}$.

2) The permutations $\Sigma, \mathrm{A}$ and $\Phi$ are given by the formulas

$$
\Sigma=\sigma_{1}^{2}, \quad \mathrm{~A}=\sigma_{2}^{2}, \quad \Phi=\sigma_{2}^{-1} \sigma_{3}^{2} \sigma_{2},
$$

where $\sigma_{1}, \sigma_{2}, \sigma_{3}$ are the generators of $\mathcal{H}_{4}$, with their action on the constellations given in (1).

We chose the term "megamap" because the term "hypermap" is already taken, while "super" usually means anti-comutative. Probably a French term "château de cartes" would be more appropriate.

Let us add some remarks concerning the interpretation of the various geometric elements of the resulting hypermap. We have already mentioned that its edges are labelled by (non-isomorphic) 4-constellations. Now let us consider a black vertex. When the fourth critical value $z$ tends to 0 and finally becomes equal to 0 , what we get is a covering with three critical values $0=z, 1$ and $\infty$, i.e., a dessin d'enfant. Combinatorially it is represented by the triple of permutations $[\sigma, \alpha, \varphi]=\left[g_{1} g_{2}, g_{3}, g_{4}\right]$. Only there is no garantee that it is connected; in the latter case it must be considered as a disjoint union of dessins. Note that the operation $\sigma_{1}$ does not change $g_{3}$ and $g_{4}$, and therefore the product $g_{1} g_{2}$ is also preserved. Thus for all the edges adjacent to a black vertex the corresponding triple $\left[g_{1} g_{2}, g_{3}, g_{4}\right]$ will be the same.

In the same way all the white vertices are labelled by the (probably disconnected) dessins $[\sigma, \alpha, \varphi]=$ $\left[g_{1}, g_{2} g_{3}, g_{4}\right]$. 
In order to find a dessin d'enfant that corresponds to the center of a face, we would like to multiply $g_{2}$ (corresponding to $z$ ) and $g_{4}$ (corresponding to $\infty$ ). But we must first transpose $g_{2}$ and $g_{3}$ by acting by $\sigma_{2}^{-1}$ and thus getting the constellation $\left[g_{1}, g_{2} g_{3} g_{2}^{-1}, g_{2}, g_{4}\right]$. If we now act on this latter 4-constellation by $\Phi$ (and then transpose once more the second and the third permutations in it via $\sigma_{2}^{-1}$ ), the resulting triple $\left[g_{1}, g_{2} g_{3} g_{2}^{-1}, g_{2} g_{4}\right]$ will not change.

\section{Examples}

Computing a megamap is not an easy task. One must not only manipulate the permutations, but verify every time if a newly obtained constellation is isomorphic to any of the previous ones. A program (in Magma language) was implemented by Nicolas Hanusse [Han97], which permitted us to compute the majority of the examples given below.

Example 4.1 Let us consider the generic rational functions of degree 3 or, in other words, the coverings corresponding to the passport

$$
[21,21,21,21]
$$

There are four non-isomorphic constellations with this passport, and they are arranged into the megamap shown in Figure 4.

Now consider the generic polynomials of degree 4 , that is, the coverings with the passport

$$
\left[21^{2}, 21^{2}, 21^{2}, 4\right] \text {. }
$$

It turns out that there are also four non-isomorphic constellations, and the corresponding megamap is the same as above!

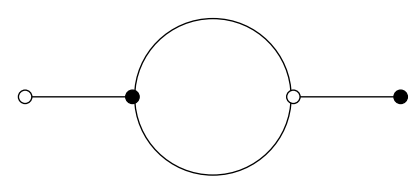

Fig. 4: A megamap which describes the "moduli space" of the generic rational functions of degree 3, and also that of the generic polynomials of degree 4

We see that this example, which is probably the simplest possible, already leads to a rather non-trivial conclusion: there exists a transformation which, for any given generic polynomial of degree 4, produces a generic rational function of degree 3 , and which is a biholomorphic bijection (the rational function becomes non-generic if and only if the polynomial taken was also non-generic). It would be interesting to find this transformation explicitly, but for the moment the author has no idea of how it could look like.

Example 4.2 For the polynomials of degree 6 with the passport $\left[321,21^{4}, 21^{4}, 6\right]$ the corresponding megamap was in fact shown in Figure 1.

For the polynomials also of degree 6 with the passport $\left[31^{3}, 21^{4}, 2^{2} 1^{2}, 6\right]$ the corresponding megamap is shown in Figure 5. 


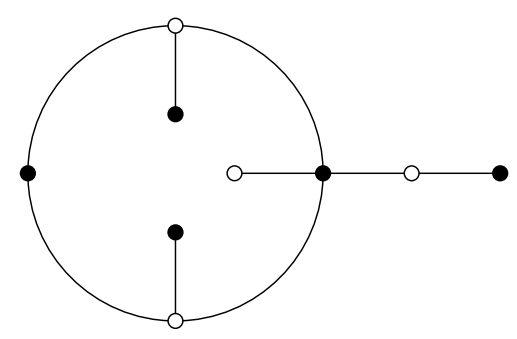

Fig. 5: A megamap for the polynomials with the passport $\left[31^{3}, 21^{4}, 2^{2} 1^{2}, 6\right]$

Example 4.3 Let us consider the polynomials of degree 7 with the passport $\left[2^{2} 1^{3}, 2^{2} 1^{3}, 2^{2} 1^{3}, 7\right]$. This example is of special interest, as this passport is one of the only three exisiting exceptional polynomial passports (see details in [JZ01]). There exist 56 non-isomorphic constellations with this passport; but this time they don't form a single orbit of the braid group. In fact, there are four orbits, of sizes 7, 7, 21, and 21 respectively. Therefore we must construct four different megamaps. It turns out that the two smaller megamaps (of size 7) are isomorphic; the corresponding picture is shown in Figure 6.

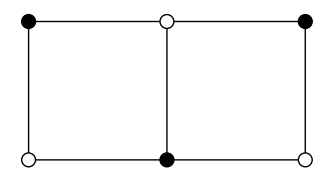

Fig. 6: A megamap representing both of the two orbits of size 7 of Example 4.3
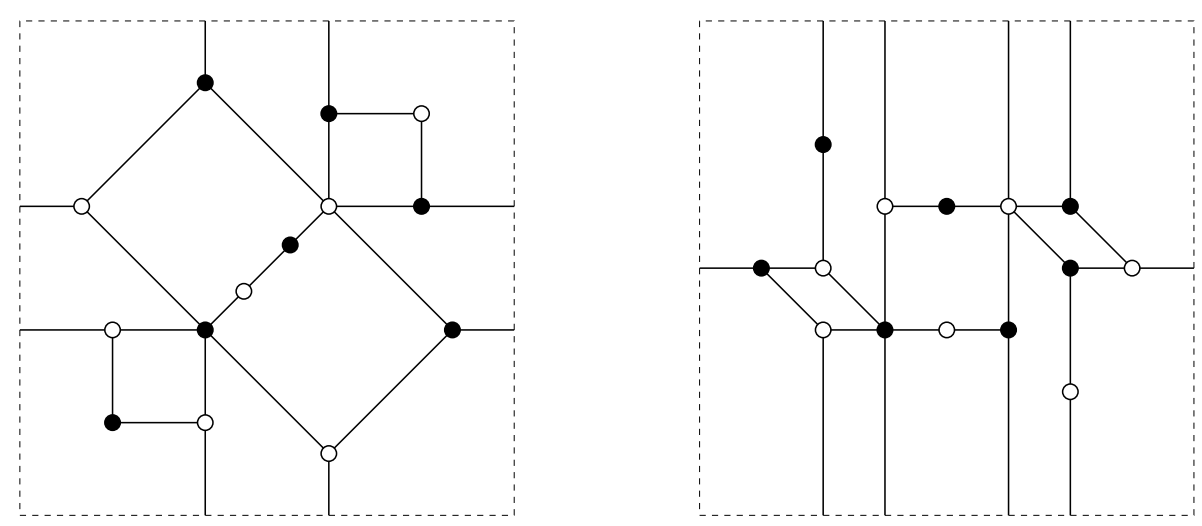

Fig. 7: Megamaps of genus 1 representing two orbits of size 21 of Example 4.3

Concerning the bigger orbits, they are not isomorphic. The easiest way to see this is to look at the picture: the corresponding megamaps are shown in Figure 7. For example, in the hypermap on the left 
there is an edge that connects two vertices of degree 2, while in the hypermap on the right such an edge does not exist.

Both dessins are of genus 1 and thus represent elliptic curves. Note that in both cases the degrees of the black vertices, of the white vertices and of the faces form the same partition $53^{4} 2^{2}$. Certainly these 'dessins d'enfants' are Galois conjugate.

Example 4.4 In this last example we supply not only combinatorial but also analytic information. Let us consider polynomials of degree $n$ with the passport $\pi=\left[(n-2) 1^{2}, 21^{n-2}, 21^{n-2}, n\right]$. The combinatorial computations give the megamap shown in Figure 8.

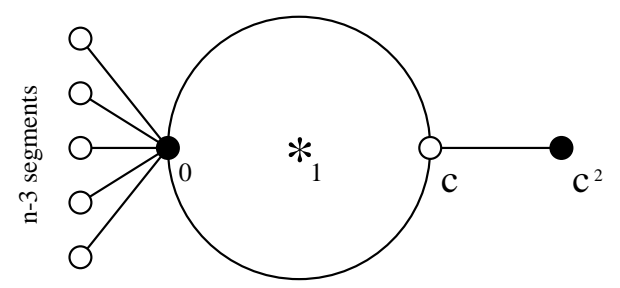

Fig. 8: A megamap of Example 4.4

This time the dessin d'enfant is simple enough, and we are able to find the corresponding Belyi function explicitly: placing the black vertex of degree $n-1$ to 0 , the center of the smaller face to 1 (the center of the outer face is placed at infinity), the white vertex of degree 3 to $c=\frac{n}{n-2}$, and the black vertex of degree 1 to $c^{2}$, we get

$$
f(t)=-\frac{1}{c^{n}} \frac{t^{n-1}\left(t-c^{2}\right)}{t-1} .
$$

(The only thing that needs to be verified is the fact that $f-1$ has indeed a root of multiplicity 3 at $c$.) But this information is not yet complete. We would also like to find a representation of the polynomials of degree $n$ with the passport $\pi$ given above. The following formula provides the answer. Let us take a polynomial in $x$ depending also on a parameter $t$ :

$$
P_{t}(x)=\frac{1}{t-1} x^{n-2}\left[(n-1) x^{2}+(n+(n-2) t) x+(n-1) t\right] .
$$

As any polynomial, it has a critical value of multiplicity $n$ at infinity. By construction, it also has a critical point of multiplicity $n-2$ at 0 (with the corresponding critical value also equal to 0 ). What are its other critcal points and values? In order to answer this question let us compute its derivative:

$$
P_{t}^{\prime}(x)=\frac{n(n-1)}{t-1} x^{n-3}(x-1)(x-t / c) .
$$

Thus the critical points are 0,1 and $t / c$, while the corresponding critical values are $P_{t}(0)=0, P_{t}(1)=1$ and

$$
P_{t}(t / c)=-\frac{1}{c^{n}} \frac{t^{n-1}\left(t-c^{2}\right)}{t-1} .
$$

The latter expression coincides with the Belyi function given in (3). 


\section{Acknowledgements}

The author is grateful to N. Adrianov, J. Bétréma, D. Bouya, J.-M. Couveignes, N. Hanusse, G. Jones and G. Shabat for fruitful discussions.

\section{References}

[Bel79] Belyй G. V. On Galois extensions of a maximal cyclotomic field. - Math. USSR Izvestija, 1980, vol. 14, no. 2, 247-256. (Original Russian paper: Izv. Acad. Nauk SSSR, ser. mat., 1979, vol. 43, no. 2, 269-276.)

[Bir74] Birman J. S. Braids, Links and Mapping Class Groups. - Princeton University press, 1974 (Annals of Math. Studies, vol. 82).

[CM92] Cori R., Machì A. Maps, hypermaps and their automorphisms: a survey, I, II, III. - Expositiones Mathematicae, 1992, vol. 10, 403-427, 429-447, 449-467.

[Cou97] Couveignes J.-M. Quelques revêtements définis sur Q. - Manuscripta Math., 1997, vol. 92, no. 4, 409-445.

[Fri90] Fried M. D. Arithmetic of 3 an 4 branch point covers. A bridge provided by noncongruence subgroups of $S L_{2}(\mathbf{Z})$. - In "Séminaire de Théorie des Nombres, Paris, 1987-88", Birkhäuser, "Progress in Mathematics", vol. 81, 1990, 77-117.

[Gra96] Granboulan L. Construction d'une extension régulière de $\mathbf{Q}(T)$ de groupe de Galois $M_{24}$. Experimental Math., 1996, vol. 5, no. 1, 3-14.

[Gro84] Grothendieck A. Esquisse d'un programme (1984). - In: Schneps L., Lochak P., eds. “Geometric Galois Action. Vol. 1: Around Grothendieck's Esquisse d'un Programme”, London Math. Soc. Lecture Notes Series, vol. 242, Cambridge Univ. Press, 1997, 5-48. (English translation: "Scketch of a programme", the same volume, p. 243-284.)

[Han97] Hanusse N. Cartes, constellations et groupes : questions algoritmiques. - Ph.D. thesis, Bordeaux, 1997.

[Hur1891] Hurwitz A. Über Riemann'sche Fläche mit gegebenen Verzweigungspunkten. - Math. Ann., 1891, vol. 39, 1-61.

[JZ01] Jones G. A., Zvonkin A. K. Orbits of braid groups on cacti. - Submitted to The Moscow Mathematical Journal.

[Sch94] Schneps L. (ed.) The Grothendieck Theory of Dessins d'Enfants. - London Math. Soc. Lecture Notes Series, vol. 200, Cambridge Univ. Press, 1994.

[Vö196] Völklein H. Groups as Galois Groups. An Introduction. - Cambridge University Press, 1996 ("Cambridge Studies in Advanced Mathematics", vol. 53). 
